\title{
The effect of density fluctuations on electron cyclotron beam broadening and implications for ITER
}

\author{
A. Snicker ${ }^{1}$, E. Poli ${ }^{1}$, O. Maj ${ }^{1}$, L. Guidi ${ }^{1}$, A. Köhn ${ }^{1}$, H. Weber ${ }^{1}$, \\ M. Henderson ${ }^{2}$, G. Saibene ${ }^{3}$ \\ ${ }^{1}$ Max-Planck-Institut für plasmaphysik, Garching, Germany \\ ${ }^{2}$ ITER Organization, 13108 St Paul lez Durance, France \\ ${ }^{3}$ Fusion for Energy, C/Josep Pla 2, Torres Diagonal Litoral-B3, E-08019 Barcelona, \\ Spain \\ E-mail: antti.snicker@ipp.mpg.de
}

\begin{abstract}
We present state-of-the-art computations of propagation and absorption of electron cyclotron waves, retaining the effects of scattering due to electron density fluctuations. In ITER, injected microwaves are foreseen to suppress neoclassical tearing modes (NTMs) by driving current at the $q=2$ and $q=3 / 2$ resonant surfaces. Scattering of the beam can spoil the good localization of the absorption and thus impair NTM control capabilities. A novel tool, the WKBeam code, has been employed here in order to investigate this issue. The code is a Monte Carlo solver for the wave kinetic equation and retains diffraction, full axisymmetric tokamak geometry, determination of the absorption profile and an integral form of the scattering operator which describes the effects of turbulent density fluctuations within the limits of the Born scattering approximation. The approach has been benchmarked against the paraxial WKB code TORBEAM and the full-wave code IPF-FDMC. In particular, the Born approximation is found to be valid for ITER parameters. In this paper, we show that the transport in ITER is diffusive unlike in present experiments, thus causing up to a factor of 2 to 4 broadening in the absorption profile. However, the broadening depends strongly on the turbulence model assumed for the density fluctuations, which still has large uncertainties.
\end{abstract}

\section{Introduction}

In order to obtain stable operation of a tokamak reactor, instabilities endangering the confinement or performance of the plasma need to be controlled, preferably in automatic manner. One of such instabilities is the neoclassical tearing mode (NTM) [1]. In existing machines, NTM mitigation and suppression has been successfully obtained using electron-cyclotron current drive (ECCD) to replace the missing bootstrap current inside the magnetic island [2]. The effect relies on the ability to focus and steer electroncyclotron (EC) beams by mirrors, thus enabling accurate localization of the driven 
current profile. This demands accurate knowledge of the equilibrium, i.e. the location of the resonance surface where the beam will be aimed. By controlling injection angles (poloidal/toroidal), the necessary amount of current can be driven inside the NTM island to stabilize it. Correspondingly, effort has been put to predict whether such a control system is plausible for ITER [3, 4]. Based on these analyses, the EC upper launcher (UL) has been assigned mainly to control NTMs $[5,6,7,8]$. During the development of the UL EC system, the physics phenomena possibly jeopardizing the stabilization of NTMs have been identified [9]. Out of these phenomena, the most important and still difficult to quantify issue is the beam broadening by the scattering due to turbulent density fluctuations and this paper aims to give more insight in this area using state-of-the-art modeling.

When the injected microwave beam propagates in the plasma, density fluctuations act as random lens and refract the beam. The effect of these fluctuations on beam broadening depends on the fluctuation characteristics and the relative position of the fluctuations to the resonance surface where the current is to be driven. Since these factors are particularly favorable for existing machines, beam broadening is fairly difficult to measure. However, some evidence of such of an effect has been recently reported [10] and more effort is put to further investigate the issue experimentally. Simultaneously, modeling efforts have been reported to study this effect $[11,12]$.

Unfortunately, most of the modeling tools like beam or ray tracing codes are not applicable in the presence of small scale fluctuations. The current work aims to overcome these issues by presenting simulations carried out using the wave kinetic approach. The turbulent fluctuations can be assumed to be frozen in the plasma - characteristic velocities of turbulent structures are much smaller than the beam group velocity and at the same time the beam power is on over several turbulence turnover times. Therefore, the effect of turbulence can be studied by averaging over large number of realizations of the turbulent fields or by means of the formalism of the wave kinetic equation which allows us to derive an averaged scattering operator describing directly the average effect of turbulence on the beam. While the former strategy is usually adopted by full-wave codes [13], the latter is the choice of WKBeam code [14] used in this work. The main advantage of this reduced model is the reduced cost of the computations, while keeping the key physics in the model, namely, beam diffraction, scattering (adopting the Born approximation as described below), absorption and full axisymmetric tokamak geometry. The scattering operator in general includes the possible scattering from the launched mode to secondary mode [15]. In the case of ITER, power converted from first-harmonic ordinary to extraordinary mode is reflected at the corresponding cut-off at the plasma boundary, thus causing stray radiation. However, cross-polarization scattering does not lead per se to beam broadening and will not be discussed in this paper.

After this introduction, in Section 2, we present the theory behind the WKBeam code and give a short overview of the scattering operator. Verification and benchmarking studies are briefly discussed to build trust on the implementation of the theory. We will present the model to be used for density fluctuations. The underlying radiation transport 
process is discussed in detail. For focused beams, the impact of turbulent fluctuations on beam broadening increases with machine size. The effect is predicted to be large for ITER, but a measurement of the effect is difficult on existing machines. Moreover, depending on the parameters, the transport can be diffusive or superdiffusive. In present-day experiments like ASDEX Upgrade, the transport tend to be superdiffusive, which leads to the formation of elevated tails in the absorption profile but a limited reduction of the peak absorption. On the other hand, the same analysis tells that ITER lies in the diffusive regime. In Section 3, we will introduce the main inputs of the studied 15 MA ITER H-mode scenario, together with the beam and mirror parameters. We will show the absorption profiles of the beams with and without the fluctuations and also present relevant scans over the input quantities that cannot be realistically extrapolated to ITER. Results show up to a factor of 2.5-4.5 broadening in the deposition profiles compared to the case without fluctuations. In Section 4, we discuss the implications of our results to NTM control in ITER.

\section{Theoretical background and numerical models}

\subsection{Wave kinetic equation}

The wave kinetic equation with random fluctuations was originally formulated in [16] adapting a statistical approach proposed by Karal and Keller [17] in combination with Weyl-symbol calculus for the phase-space representation of the wave field [18]. The specific form of the wave kinetic equation relevant to coherent wave beams and implemented in WKBeam [14] is usually written in coordinates normalized to the scale $L$ of the equilibrium plasma profiles so that the semiclassical parameter $\kappa=\omega L / c=k_{0} L$ appears explicitly in the theory; here $\omega$ is the launched frequency, $c$ the speed of light in free space, and $k_{0}=\omega / c$ is the wave number in free space. It is important to note that the scale length $L$ refers to the averaged plasma equilibrium which does not include turbulent fluctuations. Hence the parameter $\kappa$ is large even in presence of short-scale fluctuations and the semiclassical limit $\kappa \rightarrow+\infty$ can be considered, yet not directly on the solution of Maxwell's equations for the wave field, but rather on the averaged wave energy density, which is represented by the averaged Wigner function. The wave kinetic equation describes the statistically averaged Wigner function of the beam in the limit $\kappa \rightarrow+\infty$. We refer to Weber et al [14] for a sketch of the derivation. Here instead we start from the wave kinetic equation written in physical coordinates as appropriate for the applications. It reads

$$
\begin{aligned}
\left\{H_{\alpha}, w_{\alpha}\right\} & =-2 k_{0} \gamma_{\alpha} w_{\alpha}+\sum_{\beta} S_{\alpha \beta}\left(w_{\alpha}, w_{\beta}\right) \\
H_{\alpha} w_{\alpha} & =0
\end{aligned}
$$

where, for each propagation mode labeled by Greek indices, $H_{\alpha}$ is the Hamiltonian of standard ray-tracing theory [19,20], $\gamma_{\alpha}$ the absorption coefficient, $S_{\alpha \beta}$ the scattering operator to be discussed below in more detail. The unknown is the statistically averaged 
Wigner function $w_{\alpha}=w_{\alpha}(x, N)$ which depends on the spatial coordinates $x$ and the refractive index $N$. The left-hand side is written in terms of canonical Poisson brackets on the $(x, N)$ phase space, given by

$$
\left\{H_{\alpha}, w_{\alpha}\right\}=\nabla_{N} H_{\alpha} \cdot \nabla_{x} w_{\alpha}-\nabla_{x} H_{\alpha} \cdot \nabla_{N} w_{\alpha} .
$$

One should notice that the wave kinetic equation has the form of a steady-state kinetic equation with an additional constraint. The Wigner function can be associated to the electric field energy density. It has an analogous role to distribution function of the kinetic theory as we can derive integral expressions over the Wigner function for any quantity that can be written in terms of $|E|^{2}$, e.g., the absorption profile. The constraint merely means that there is no energy on phase-space points that do not satisfy the dispersion relation of the mode, $H_{\alpha}=0$. Further details on the Monte Carlo solution of the wave kinetic equation and on how to construct such integrals of Wigner function are given in [14].

In WKBeam, the Hamiltonians $H_{\alpha}=e_{\alpha}^{*} D e_{\alpha}$ are computed from the cold-plasma dispersion tensor $D_{i j}=N^{2} \delta_{i j}-N_{i} N_{j}-\varepsilon_{i j}$ with $\varepsilon=\left(\varepsilon_{i j}\right)$ being the cold-plasma dielectric tensor [21] and $e_{\alpha}(x, N)$ the polarization unit vector of the mode $\alpha$; in a cold plasma, there are two propagation modes, namely the ordinary mode (O-mode, $\alpha=\mathrm{O}$ ) and the extra-ordinary mode (X-mode, $\alpha=\mathrm{X}$ ).

The absorption coefficient $\gamma_{\alpha}$, on the other hand, is computed from the hot-plasma dielectric tensor accounting for relativistic electron-cyclotron resonance interaction. More specifically, one can write $\gamma_{\alpha}=\operatorname{Im}(N) \cdot \nabla_{N} H_{\alpha}$ where $\operatorname{Im}(N)$ is the imaginary part of the refractive index vector which is computed in the WKBeam code by the same routines used in the complex-geometrical-optics code GRAY [22] and the paraxial WKB code TORBEAM [23].

The general form of the scattering operator includes cross-polarization scattering terms from mode $\alpha$ to mode $\beta$ and is given by

$$
\begin{aligned}
S_{\alpha \beta}\left(w_{\alpha}, w_{\beta}\right)(x, N)=\int\left[\sigma_{\beta \alpha}(\right. & \left.x, N^{\prime}, N\right) w_{\beta}\left(x, N^{\prime}\right) \\
& \left.-\sigma_{\alpha \beta}\left(x, N, N^{\prime}\right) w_{\alpha}(x, N)\right] \mathrm{d} N^{\prime},
\end{aligned}
$$

where the integration is over the refractive index space. Here the scattering cross-section

$$
\begin{aligned}
\sigma_{\alpha \beta}\left(x, N, N^{\prime}\right)=2 \pi k_{0} \mid e_{\alpha}^{*}(x, N) & \left.(\mathbb{1}-\varepsilon) e_{\beta}\left(x, N^{\prime}\right)\right|^{2} \\
& \times\left(\frac{k_{0}}{2 \pi}\right)^{3} \Gamma\left(x, N-N^{\prime}\right) \delta\left(H_{\beta}\left(x, N^{\prime}\right)\right),
\end{aligned}
$$

describes a momentum-preserving three-wave interaction process in which a mode $\alpha$ carrying refractive index $N$ interacts with turbulence exchanging momentum $\Delta N$ and thus scattering into a mode $\beta$ with refractive index $N^{\prime}$, with momentum conservation $N-N^{\prime}=\Delta N$. The spatial position is not changed in the scattering event. The variation in momentum $\Delta N$ is provided by the turbulence spectrum $\Gamma$ which is rigorously defined as the Wigner transform of the two-point correlation function of the relative electron density fluctuations $\delta n_{e}$, namely,

$$
\Gamma(x, N)=\frac{1}{n_{e}(x)^{2}} \int e^{-i k_{0} N \cdot s} \mathbb{E}\left(\delta n_{e}(x+s / 2) \delta n_{e}(x-s / 2)\right) d s,
$$


where $n_{e}$ is the average electron density profile of the plasma.

In the WKBeam code, the two-point correlation function of the electron density fluctuations is a critical input quantity. Such information is usually not easily recovered, neither from experimental data nor from simulations. Therefore we make use of a model which is based on the current theoretical understanding of turbulence in a magnetized plasma, thus reducing the required inputs to quantities that can be inferred from available data. The model used in this work amounts to

$$
\frac{1}{n_{e}(x)^{2}} \mathbb{E}\left(\delta n_{e}(x+s / 2) \delta n_{e}(x-s / 2)\right)=F(x)^{2} e^{-\frac{1}{2} s \cdot A(x) s},
$$

where $F(x)$ is a scalar function accounting for the spatial localization of turbulent density fluctuations and

$$
A_{i j}(x)=L_{\perp}^{-2}\left(\delta_{i j}-b_{i}(x) b_{j}(x)\right)+L_{\|}^{-2} b_{i}(x) b_{j}(x),
$$

is a tensor accounting for the anisotropy of turbulence in magnetized plasmas, $L_{\perp}$ and $L_{\|}$being the correlation lengths in perpendicular and parallel directions with respect to the unit vector $b$ of the equilibrium magnetic field. The input parameters for the code are thus reduced to the function $F$, which is usually specified on the poloidal plane of the tokamak and the two correlation lengths, cf. section 2.3 below. The function $F$ in particular can be identified with the root-mean-square density fluctuations: In fact, we have, upon setting $s=0$,

$$
F(x)=\sqrt{\frac{\mathbb{E}\left(\delta n_{e}(x)^{2}\right)}{n_{e}(x)^{2}}} \equiv\left[\frac{\delta n_{e}}{n_{e}}\right]_{\mathrm{rms}}(x),
$$

and the average here is a statistical average (no spatial averaging should be performed). A further advantage of this model is that the integral in spectrum $\Gamma$ can be computed analytically.

\subsection{An estimate of the effect of density fluctuations}

The effect of the scattering operator on the solution of the wave kinetic equation can be rather complicated and it is in general not just a diffusion process. We can in fact distinguish two radically different regimes, namely, a superdiffusive regime in which scattering deforms the tails of the wave energy distribution only with little consequences for the quality of the beam, and a diffusive regime in which the whole wave energy distribution spreads with a significant broadening of the beam. An indication on the scattering regime can be obtained from the total scattering cross-section

$$
\Sigma_{\alpha}(x, N)=\sum_{\beta} \int \sigma_{\alpha \beta}\left(x, N, N^{\prime}\right) \mathrm{d} N^{\prime},
$$

which is the average number of scattering events per unit of propagation length for the mode $\alpha$ and near the phase-space point $(x, N)$. If $\Delta \ell$ is the distance traveled by a beam in the turbulent plasma, then

$$
\lambda_{\alpha}=\left\langle\Sigma_{\alpha}\right\rangle \Delta \ell
$$


where $\left\langle\Sigma_{\alpha}\right\rangle$ is the average of $\Sigma_{\alpha}(x, N)$ along the beam path (reference ray), gives an estimate of the average number of scattering events for that beam. For $\lambda_{\alpha} \gg 1$ we expect to have diffusive solutions with significant broadening of the beam. It is therefore useful to have simple estimates for such a parameter.

We begin with the analytical calculation of the spectrum $\Gamma$, which in view of equation (7) amounts to

$$
\left(\frac{k_{0}}{2 \pi}\right)^{3} \Gamma\left(k_{0}, x, N\right)=\frac{F(x)^{2}}{(2 \pi)^{3 / 2} \sigma_{\perp}^{2} \sigma_{\|}} \exp \left(-\frac{N_{\perp}^{2}}{2 \sigma_{\perp}^{2}}-\frac{N_{\|}^{2}}{2 \sigma_{\|}^{2}}\right),
$$

where we have introduced the parameters $\sigma_{\perp}=\left(k_{0} L_{\perp}\right)^{-1}$ and $\sigma_{\|}=\left(k_{0} L_{\|}\right)^{-1}$ that play the role of spectral widths and cylindrical coordinates defined by

$$
N=N_{\|} b(x)+N_{\perp}\left(e_{1}(x) \cos \phi_{N}+e_{2}(x) \sin \phi_{N}\right),
$$

with $\left(e_{1}, e_{2}, b\right)$ being an orthogonal reference frame constructed around the unit vector $b$ of the local magnetic field.

Turbulence in a magnetized plasma is well within the limit $k_{0} L_{\|} \gg 1$ both because of the high frequency of the wave and the flute-mode character of turbulence. Hence we can consider the limit $\sigma_{\|} \rightarrow 0^{+}$, for which one has

$$
\left(\frac{k_{0}}{2 \pi}\right)^{3} \Gamma\left(k_{0}, x, N\right) \rightarrow \frac{F(x)^{2}}{2 \pi \sigma_{\perp}^{2}} \exp \left[-\frac{N_{\perp}^{2}}{2 \sigma_{\perp}^{2}}\right] \delta\left(N_{\|}\right) .
$$

The presence of the Dirac's delta-function together with two key properties of the coldplasma dispersion tensor allow us to compute the total cross-section exactly. The key properties are: (i) The dispersion relation $H_{\alpha}(x, N)=0$ corresponds to $N_{\perp}=n_{\alpha}\left(x, N_{\|}\right)$ with $n_{\alpha}$ a known function; (ii) The dispersion tensor and thus the polarization vectors $e_{\alpha}(x, N)$ depend only on $N_{\perp}, N_{\|}$and not on the polar angle $\phi_{N}$. Particularly (i) implies

$$
\delta\left(H_{\beta}\left(x, N^{\prime}\right)\right)=\left|\frac{\partial H_{\beta}}{\partial N_{\perp}}\right|^{-1} \delta\left(N_{\perp}^{\prime}-n_{\beta}\left(x, N_{\|}^{\prime}\right)\right) .
$$

In view of such properties and in the limit $\sigma_{\|} \rightarrow 0^{+}$, we obtain

$$
\Sigma_{\alpha}=\frac{k_{0} F^{2}}{\sigma_{\perp}^{2}} \sum_{\beta} M_{\alpha \beta} \int_{0}^{2 \pi} e^{-\left(n_{\alpha} n_{\beta} / \sigma_{\perp}^{2}\right)\left(1-\cos \left(\phi_{N}-\phi_{N}^{\prime}\right)\right)} d \phi_{N}^{\prime},
$$

where $\Sigma_{\alpha}$ has been evaluated "on-shell", i.e., on the dispersion surface $N_{\perp}=n_{\alpha}\left(x, N_{\|}\right)$, and we have defined the matrix of coefficients

$$
M_{\alpha \beta}=n_{\beta}\left[\frac{\left|e_{\alpha}^{*}(x, N)(\mathbb{1}-\varepsilon) e_{\alpha}\left(x, N^{\prime}\right)\right|^{2}}{\left|\partial H_{\beta}\left(x, N^{\prime}\right) / \partial N_{\perp}\right|}\right] \exp \left(-\frac{\left(n_{\alpha}-n_{\beta}\right)^{2}}{2 \sigma_{\perp}^{2}}\right),
$$

where both $n_{\alpha}$ and $n_{\beta}$ should be evaluated at $\left(x, N_{\|}\right)$, while the term in square brackets should be evaluated at $N_{\|}^{\prime}=N_{\|}$and $N_{\perp}^{\prime}=n_{\beta}\left(x, N_{\|}\right)$.

The remaining integral can be computed by means of the Jacobi-Anger expansion,

$$
e^{i z \cos \theta}=\sum_{n \in \mathbb{Z}} i^{n} J_{n}(z) e^{i n \theta},
$$


where $J_{n}(z)$ are the Bessel functions and the sum is over the set $\mathbb{Z}$ of integer numbers. We can use this identity with $z=-i n_{\alpha} n_{\beta} / \sigma_{\perp}^{2}$ and $\theta=\phi_{N}-\phi_{N}^{\prime}$ and only the term $n=0$ gives a contribution. At last we obtain

$$
\Sigma_{\alpha}=\frac{2 \pi k_{0}}{\sigma_{\perp}^{2}}\left[\frac{\delta n_{e}}{n_{e}}\right]_{\mathrm{rms}}^{2} \sum_{\beta} M_{\alpha \beta} e^{-n_{\alpha} n_{\beta} / \sigma_{\perp}^{2}} I_{0}\left(n_{\alpha} n_{\beta} / \sigma_{\perp}^{2}\right),
$$

and $I_{n}(z)=i^{-n} J_{n}(i z)$ are the modified Bessel functions of first kind and, in particular, $I_{0}$ is even so that $I_{0}\left(-n_{\alpha} n_{\beta} / \sigma_{\perp}\right)=I_{0}\left(n_{\alpha} n_{\beta} / \sigma_{\perp}\right)$.

Expression (10) is valid under the only assumption of long parallel correlation length $k_{0} L_{\|} \gg 1$ which is usually satisfied in cases of interest. If in addition, we consider the limit $\sigma_{\perp}=\left(k_{0} L_{\perp}\right)^{-1} \rightarrow 0^{+}$which is often the case for beams in the EC frequency range, the Hankel's large-argument expansion of the modified Bessel function,

$$
I_{0}(z) \sim e^{z} /(2 \pi z)^{1 / 2}
$$

yields the simpler expression

$$
\Sigma_{\alpha} \sim \sqrt{2 \pi} k_{0}\left[\frac{\delta n_{e}}{n_{e}}\right]_{\mathrm{rms}}^{2}\left[\sum_{\beta} M_{\alpha \beta}\left(n_{\alpha} n_{\beta}\right)^{-1 / 2}\right] k_{0} L_{\perp},
$$

which exhibits the linear dependence with $k_{0} L_{\perp}$.

With the aim of practical estimate of scattering, however, it is convenient to further simplify the expression for $\Sigma_{\alpha}$. In most cases the toroidal injection angles are modest, hence we consider the simple case of perpendicular propagation. In the reference frame in which the local magnetic field define the third axis and the refractive index belongs to the plane spanned by the first and third axis (Stix reference frame), perpendicular propagation implies $N=\left(N_{\perp}, 0,0\right)$. For the ordinary mode $\alpha=O$ we find

$$
e_{O}(x, N)=\left(\begin{array}{l}
0 \\
0 \\
1
\end{array}\right), \quad H_{O}=N_{\perp}^{2}-1+\frac{\omega_{p e}^{2}}{\omega^{2}}, \quad e_{O}^{*}(\mathbb{1}-\varepsilon) e_{O}=\frac{\omega_{p e}^{2}}{\omega^{2}},
$$

where $\omega_{p e}$ is the electron plasma frequency. As a result, for perpendicular propagation,

$$
M_{O O}=\frac{1}{2} \frac{\omega_{p e}^{4}}{\omega^{4}}
$$

Even under such simple conditions, the calculations for the extra-ordinary mode $\alpha=X$, although doable, yield rather cumbersome results. In fact one has

$$
e_{X}(x, N) \propto\left(\begin{array}{c}
\frac{D}{S+H_{X}} \\
i \\
0
\end{array}\right), \quad H_{X}=\frac{N_{\perp}^{2}}{2}-S+\sqrt{\frac{N_{\perp}^{4}}{4}+D^{2}},
$$

with the proportionality constant for $e_{X}$ being determined by $\left|e_{X}\right|^{2}=1$ and

where

$$
e_{X}^{*}(\mathbb{1}-\varepsilon) e_{X}=1-S \frac{S^{2}-D^{2}}{S^{2}+D^{2}},
$$

$$
S=1-\frac{\omega_{p e}^{2}}{\omega^{2}-\omega_{c e}^{2}}, \quad D=\frac{\omega_{c e}}{\omega} \frac{\omega_{p e}^{2}}{\omega^{2}-\omega_{c e}^{2}}
$$


are the standard parameters of the cold-plasma dielectric tensor and $\omega_{c e}>0$ is the electron cyclotron frequency (defined with the elementary charge so that it is a positive number). The dispersion relation $H_{X}=0$ in this case corresponds to

$$
N_{\perp}^{2}=\left(S^{2}-D^{2}\right) / S
$$

as expected, but the Hamiltonian $H_{X}$ has been computed as the eigenvalue of the dispersion tensor. Then we have

$$
M_{X X}=\frac{1}{2} \frac{\left[S^{2}+D^{2}-S\left(S^{2}-D^{2}\right)\right]^{2}}{S^{2}\left(S^{2}+D^{2}\right)} .
$$

In this specific case of perpendicular propagation, the cross-polarization terms $M_{O X}$ and $M_{X O}$ are both exactly zero, no matter how close the mode dispersion surfaces are. This is due to the block-diagonal structure of the matrix $\mathbb{1}-\varepsilon$ with the polarization $e_{O}$ and $e_{X}$ being in the subspace of the two different diagonal blocks of the matrix. The fact that modes are exactly decoupled for perpendicular propagation independently of the fluctuation level can also be inferred directly by inspection of Maxwell's equations.

The expression for $M_{X X}$ is still rather complicated for practical use. Instead of an exact calculation one could consider an easier lower bound. This can be computed on noting that the maximum and minimum values of any quadratic form $F(z)=z^{*} A z$, where $z$ is a complex vector satisfying $z^{*} z=1$ and $A$ a complex positive semi-definite Hermitian matrix, amount to the maximum and minimum eigenvalues, respectively. We can therefore obtain a bound for $e_{\alpha}^{*}(\mathbb{1}-\varepsilon) e_{\beta}$ as well as for $\partial H_{\alpha} / \partial N_{\perp}=e_{\alpha}^{*} \partial D / \partial N_{\perp} e_{\alpha}$. Since we are interested in the X-mode for perpendicular propagation, we can limit our calculation to the blocks of the matrices orthogonal to the O-mode polarization and computing the eigenvalues we obtain

$$
\frac{\omega_{p e}^{2}}{\omega\left(\omega+\omega_{c e}\right)} \leq e_{X}^{*}(\mathbb{1}-\varepsilon) e_{X} \leq \frac{\omega_{p e}^{2}}{\omega\left(\omega-\omega_{c e}\right)}, \quad 0 \leq \frac{\partial H_{X}}{\partial N_{\perp}} \leq 2 N_{\perp} .
$$

The lower bound on the derivative of $H_{X}$ cannot be used as we need $\left|\partial H_{X} / \partial N_{\perp}\right|^{-1}$. That however allows us to obtain a lower bound for $\Sigma_{X}$. In summary we have

$$
\Sigma_{O}=\sqrt{\frac{\pi}{2}} k_{0}\left[\frac{\delta n_{e}}{n_{e}}\right]_{\mathrm{rms}}^{2} \frac{\omega_{p e}^{4}}{\omega^{4}} \frac{k_{0} L_{\perp}}{n_{O}},
$$

for the ordinary mode while, with the lower bound for $M_{X X}$,

$$
\Sigma_{X} \geq \sqrt{\frac{\pi}{2}} k_{0}\left[\frac{\delta n_{e}}{n_{e}}\right]_{\mathrm{rms}}^{2} \frac{\omega_{p e}^{4}}{\omega^{2}\left(\omega+\omega_{c e}\right)^{2}} \frac{k_{0} L_{\perp}}{n_{X}},
$$

for the extra-ordinary mode, both results being valid for perpendicular propagation under the limits $k_{0} L_{\|}, k_{0} L_{\perp} \gg 1$.

A simple estimate of the parameter $\lambda_{\alpha}$ can now be readily written by using either equation (13) or (14) as relevant to the considered mode. In order to compute $\lambda_{\alpha}$ rigorously we should average $\Sigma_{\alpha}$ over the propagation path of the beam in the turbulent plasma. For simplicity however we can evaluate $\Sigma_{\alpha}$, which for perpendicular propagation depends on $x$ only, at a given point in space chosen to represent the typical value of plasma parameter in the turbulent region. Then we can write an estimate for $\lambda_{\alpha}$ which 
can be evaluated for a given tokamak scenario without need of ray- or beam-tracing calculations. That is,

$$
\lambda_{\alpha} \approx \sqrt{\frac{\pi}{2}} \frac{1}{n_{\alpha}}\left[\frac{\delta n_{e}}{n_{c}}\right]_{\mathrm{rms}}^{2}\left(k_{0}^{2} L_{\perp} \Delta \ell\right)\left\{\begin{array}{cl}
1, & \alpha=O, \\
\omega^{2} /\left(\omega+\omega_{c e}\right)^{2}, & \alpha=X,
\end{array}\right.
$$

and we have introduced the cut-off density $n_{c}=n_{e}\left(\omega / \omega_{p e}\right)^{2}$. The authors would like to remind that this chain of approximations and simplifications were made to obtain a simplified expression for the categorization of the transport regimes. In the implementation of the code, the general expression (4) is evaluated during a scattering event as described in [24].

\subsection{Model for density fluctuations}

The turbulence model employed in WKBeam simulations consists in a model profile for the function $F$ defined in equation (7), which is the root-mean-square relative electrondensity fluctuations. We model $F$ as a function of the radial coordinate $\rho_{p}=\sqrt{\psi_{p}}$, where $\psi_{p}$ is the normalized poloidal flux $\left(\psi_{p}=0\right.$ and $\psi_{p}=1$ corresponds to magnetic axis and the separatrix, respectively), and the geometrical poloidal angle $\theta$. At first we shall assume that $F$ is a purely radial profile, i.e.,

$$
F\left(\rho_{p}, \theta\right)=F_{r}\left(\rho_{p}\right),
$$

with $F_{r}$ depending only on the radial coordinate $\rho_{p}$. The specific profile used for $F_{r}$ was selected based on the experimental work in $[25,26]$ and it is shown in Figure 1. It is a piecewise defined function combining core region until $\rho_{p}=0.97$, a (linear) ramp-up region at $\rho_{p}=0.97-1.0$ and a constant edge region at $\rho_{p}>1.0$. The core value in $\mathrm{H}$-modes is a few percent (and has a negligible effect on the beam broadening), here it is chosen to be $2 \%$. While the edge level dominates the beam broadening and here it was selected to be $20 \%$. A Gaussian centered at $\rho_{p}=1$ is shown for comparison, as it has been used in the earlier works $[12,11]$. As this extrapolation from current experiments to ITER is definitely uncertain, a scan for the edge value is presented in section 3.3. The correlation length was assumed to scale like $L_{c} \approx 5-10 \rho_{s}$, where $\rho_{s}$ is the sound gyroradius. The authors are familiar that this scaling might break down when going from open to closed field lines. As the temperature of the plasma is a radial function, so is the expected correlation length. In this paper, however, a constant value of the correlation length was used as the main effect of the fluctuations can be isolated to a radial domain close to the edge (we note, however, that the theory allows a radial dependency on the correlation length). Using ITER parameters, the correlation length in the studied scenario at the location of interest is $1-2 \mathrm{~cm}$. Consequently, a value of 2 $\mathrm{cm}$ was used and a scan around this value is presented in 3.3.

In the plasma core, the turbulence exhibits strong ballooning structure leading to poloidal dependency in the density fluctuation amplitude. In general this ballooning behavior can be modeled using

$$
F\left(\rho_{p}, \theta\right)=F_{r}\left(\rho_{p}\right) \frac{1}{2}(1+\cos (\theta)),
$$




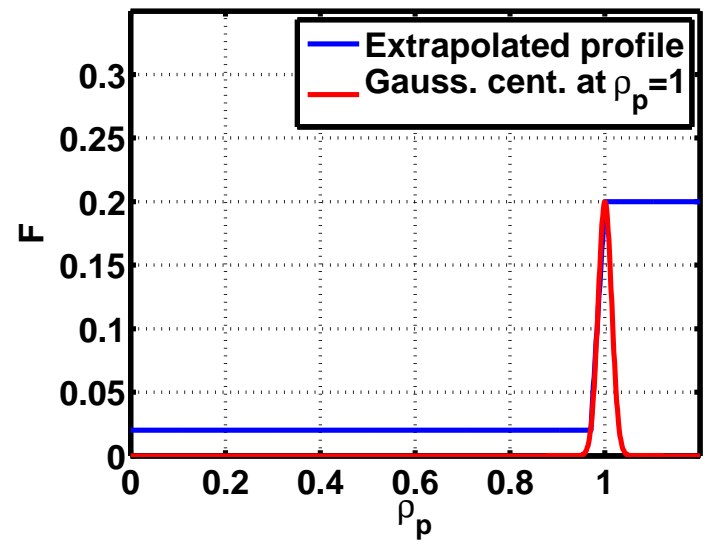

Figure 1. Radial function of the density fluctuation amplitude. Extrapolation from experimental H-mode profiles and a Gaussian profile centered at the separatrix are illustrated.

where $\theta$ is the geometrical poloidal angle. For ITER, this ballooning leads to the shape illustrated in Figure 2. In this figure the radial function shown in Figure 1 was used. At the location where the UL EC beam enters the plasma, the value of the fluctuation amplitude is roughly half of the midplane value. However, only very marginal amount of information exists about the ballooning in open field lines either experimentally or theoretically $[27,28,29]$, and even these observations are often of limited interest for us (limiter plasmas, L-mode). To compensate for these uncertainties, a scan over different fluctuation amplitude levels is performed, as detailed in the following sections.

\subsection{Verification and validation studies}

To ensure that the implementation of the theory is handled properly, several benchmarks have been carried out. These included a benchmark study without the fluctuations against well benchmarked and validated TORBEAM code [23]. Several different scenarios were studied, including various ASDEX Upgrade discharges and ITER. These cases involve variation in kinetic profiles, magnetic fields and also injection angles. Both the beam shape and absorption profiles were compared. In most of the cases, for which an ASDEX Upgrade example is shown in figure 3 and ITER examples in figures 4 and 5 , both the beam and the profiles are extremely close to each other. However, some discrepancy in the absorption profiles were observed in particular for large toroidal injection angles like shown in 5. The reason for these discrepancies is thought to be a combination of physical differences between the codes (TORBEAM adopts the paraxial approximation, so that all the relevant information is computed on the central ray only) and the projection to flux surfaces necessary in the TORBEAM side when constructing the absorption profile.

Furthermore, the code was benchmarked against full-wave code IPF-FDMC [30]. In these studies, the effect of fluctuations on the beam shape was studied under ITERrelevant conditions, i.e. $n_{e} / n_{c} \approx 0.2$. The main goal of this benchmark was to find 


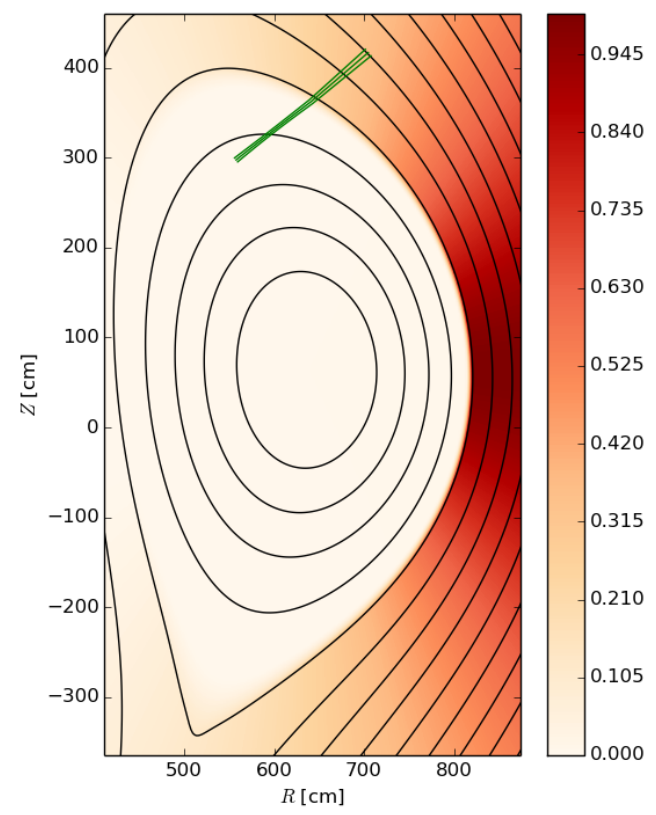

Figure 2. Ballooning of the density fluctuation using equation (16). The amplitude of the fluctuations is normalized to peak value. Note that at the region where the microwave beam enters the plasma, the fluctuation amplitude is roughly half that of the midplane value.

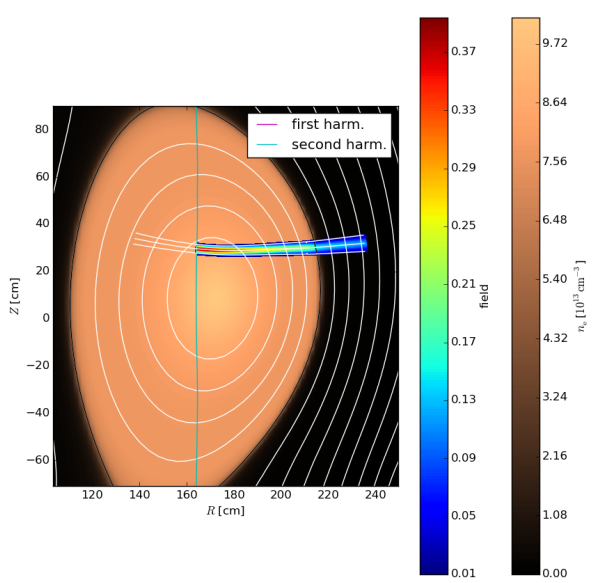

a)

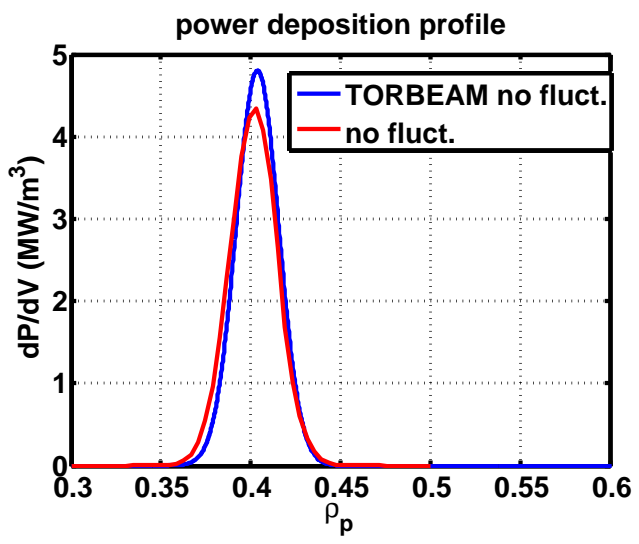

Figure 3. Comparison of the TORBEAM EC beam and WKBeam beam a) and deposition profile b) in ASDEX Upgrade discharge \# 25485. The beams are on top of each other, deposition profiles are not identical. Perpendicular injection with zero toroidal angle. 


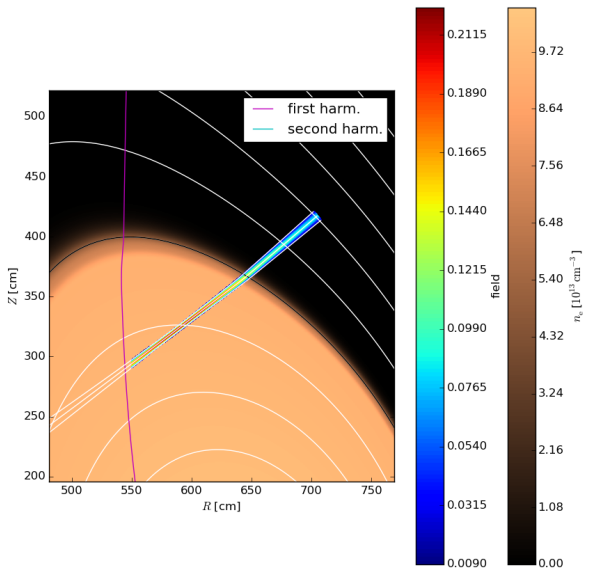

a)

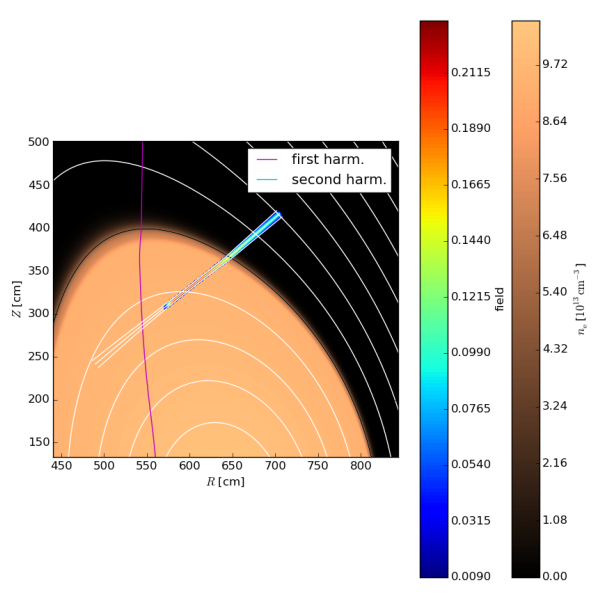

a) b)

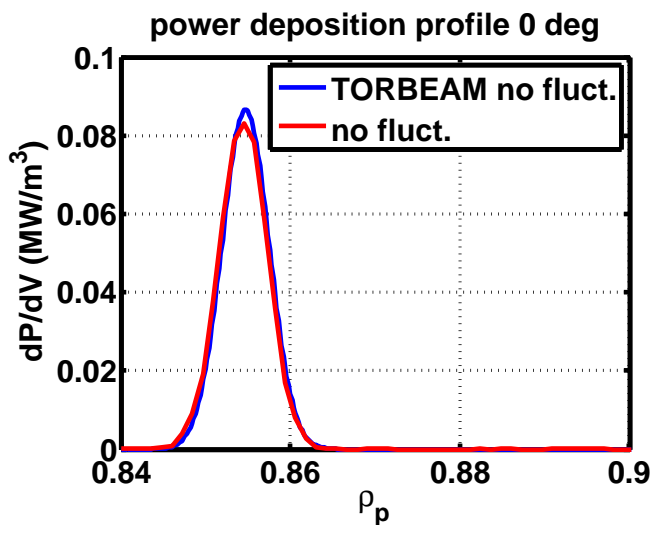

Figure 4. Comparison of the TORBEAM EC beam and WKBeam beam a) and deposition profile b) in ITER. The beams are on top of each other, deposition profiles are on top of each other. This was carried out for a perpendicular beam injection.

)

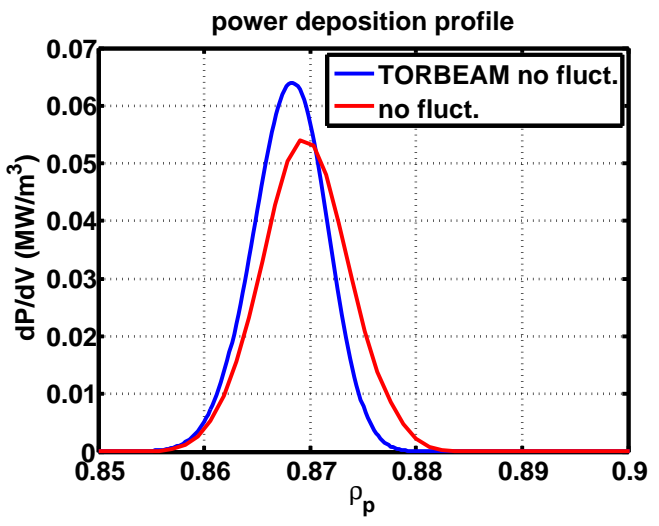

Figure 5. Comparison of the TORBEAM EC beam and WKBeam beam a) and deposition profile b) in ITER. The beams are on top of each other, deposition profiles are similar. This was carried out for nominal toroidal injection angle of 20 degrees.

the threshold above which the Born approximation employed in WKBeam breaks down (details will be presented in a separate publication). This threshold is expected to be a function of the fluctuation amplitude and, therefore, a scan against this variable was carried out until the codes disagreed. The main result is that the WKBeam method agrees well with the full-wave calculations in the levels of fluctuations amplitudes expected in ITER, i.e. $\delta n_{e} / n_{e}<40 \%$ as shown in Fig. 6. Therefore the basic 
physics assumption upon which the WKBeam code relies appears valid under ITER-like conditions.

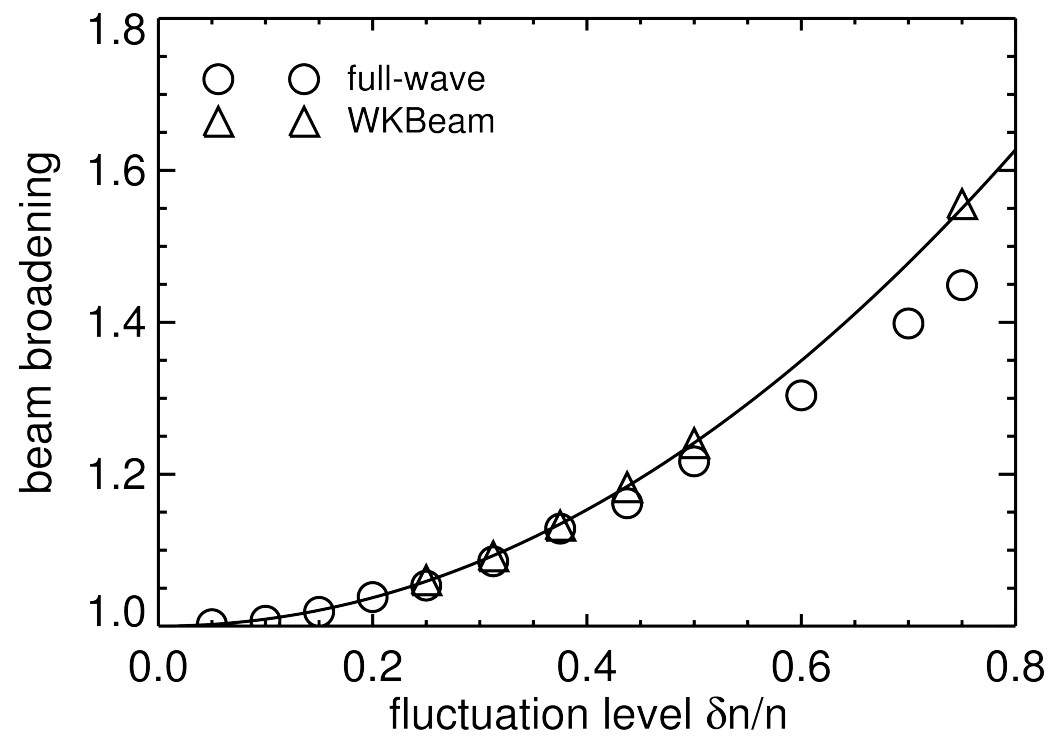

Figure 6. Demonstration of breaking down of the Born approximation using ITER relevant parameters. Full-wave and WKBeam codes agree well for fluctuation amplitudes $\delta n_{e} / n_{e}<40 \%$.

\subsection{Transport regimes - comparison of AUG and ITER}

As an example of present-day experiment, we take ASDEX Upgrade discharge \#25485. There the EC beam was in X-mode polarization with a frequency of $140 \mathrm{GHz}$. Using the information of the plasma quantities, we may construct a 2D map of the scattering parameter in eq. (15) as a function of $k_{0} L_{\perp} \Delta \ell$ and $\delta n_{e} / n_{c}$ as motivated by this equation. For this analysis, we have set a Gaussian layer around the separatrix, as illustrated in Fig. 1 and calculated the distance $s$ the beam travels in this layer. The nominal values for the correlation length was $0.4 \mathrm{~cm}$ for AUG and $2 \mathrm{~cm}$ for ITER and the nominal amplitude was $\delta n_{e} / n_{e}=10 \%$. In Fig. 7 we show the $\lambda=1$ curve together with the nominal values of $\lambda$ for the parameters discussed above. Clearly ITER is well in the diffusive regime, while AUG nominal values are close to boundary but on the superdiffusive side.

For illustrative purposes, we considered also modified cases with $L_{\perp}=2 \mathrm{~cm}$ and $\delta n_{e} / n_{e}=20 \%$ for AUG and $L_{\perp}=0.2 \mathrm{~cm}$ for ITER. With these parameters, the scattering parameter ends up clearly on the diffusive side in the AUG case and close to the boundary in ITER case. As the derivation of the scattering parameter includes several approximations, the boundary cannot be considered to be sharp. Moreover, the real transport, within the limits of our model, can be obtained by running the code 
and it was done for the two cases discussed above. The resulting statistically averaged electric energy density $\propto \mathbb{E}\left(|E|^{2}\right)$ after the fluctuation layer as a function of a distance along the perpendicular distance to the EC beam is illustrated in 8 and 9 . The diffusive beam shape in both cases can accurately fitted with a Gaussian while the superdiffusive case has characteristic high tails, that suggest a Kappa or Lévy distribution [31, 32]. To further illustrate this, we fitted a generalized Cauchy distribution with

$$
E_{\text {fit }}^{2}(x)=\frac{a^{2}}{\left(x^{2}+b^{2}\right)^{c}},
$$

where $a, b$ and $c$ are free parameters of the fit. Using the beam shape coming from WKBeam to generate the least-square fit, $c$ has a value of $2.18 \pm 0.20$ (95\% confidence level), which is very close to Cauchy distribution $(c=2)$. We have also counted the number of scattering events along each ray. In the diffusive regime, almost all rays ( $>99 \%$ ) have scattered at least once and most of them several times, whilst in the superdiffusive regime only a fraction of the rays undergo a scattering event (depends on how deep in the superdiffusive regime one lies, but here for example roughly $20 \%$ ) and having more than one event for a ray is very unlikely. Last remark is the difference between the superdiffusive beam shapes of ITER and AUG examples, in ITER the high tails are more visible than in the AUG case. This can be explained by the fact that in AUG case the fluctuation layer is so short, and the scattering probability correspondingly low, that not enough scattering events occur to raise the tails, and the original Gaussian beam shape is retained rather well.

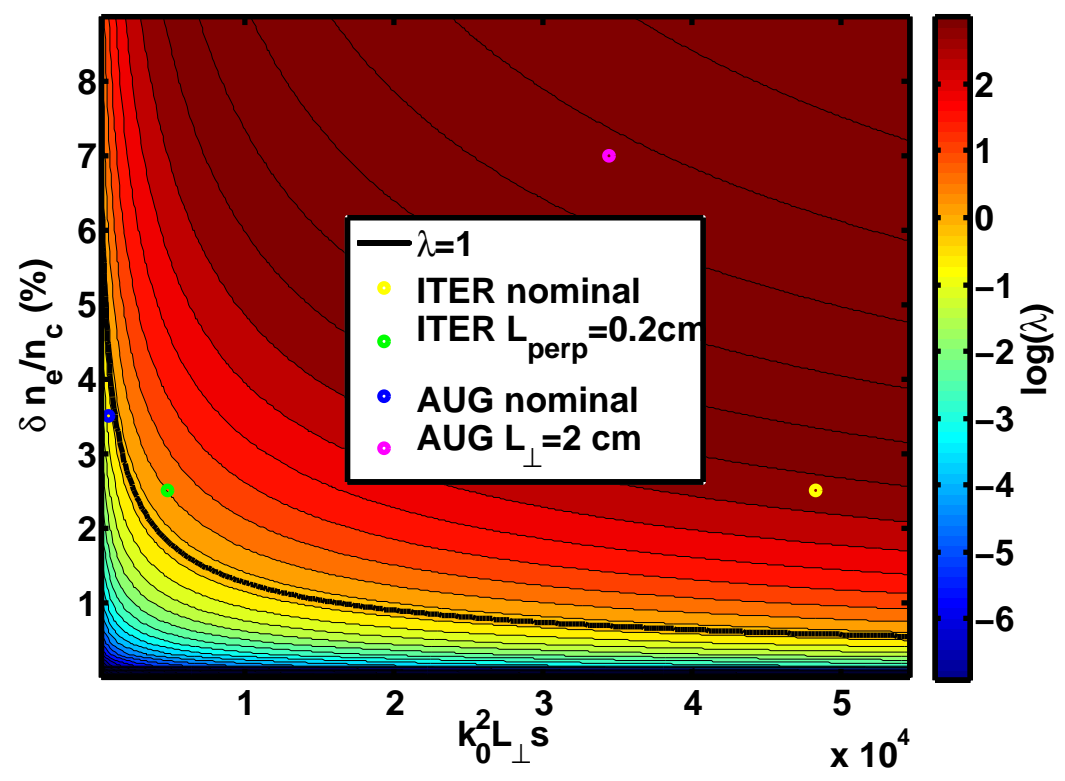

Figure 7. Transport regimes in AUG and ITER. Everything below $\lambda=1$, like AUG nominal parameters, is superdiffusive and above, like ITER and AUG modified parameters, is diffusive. 


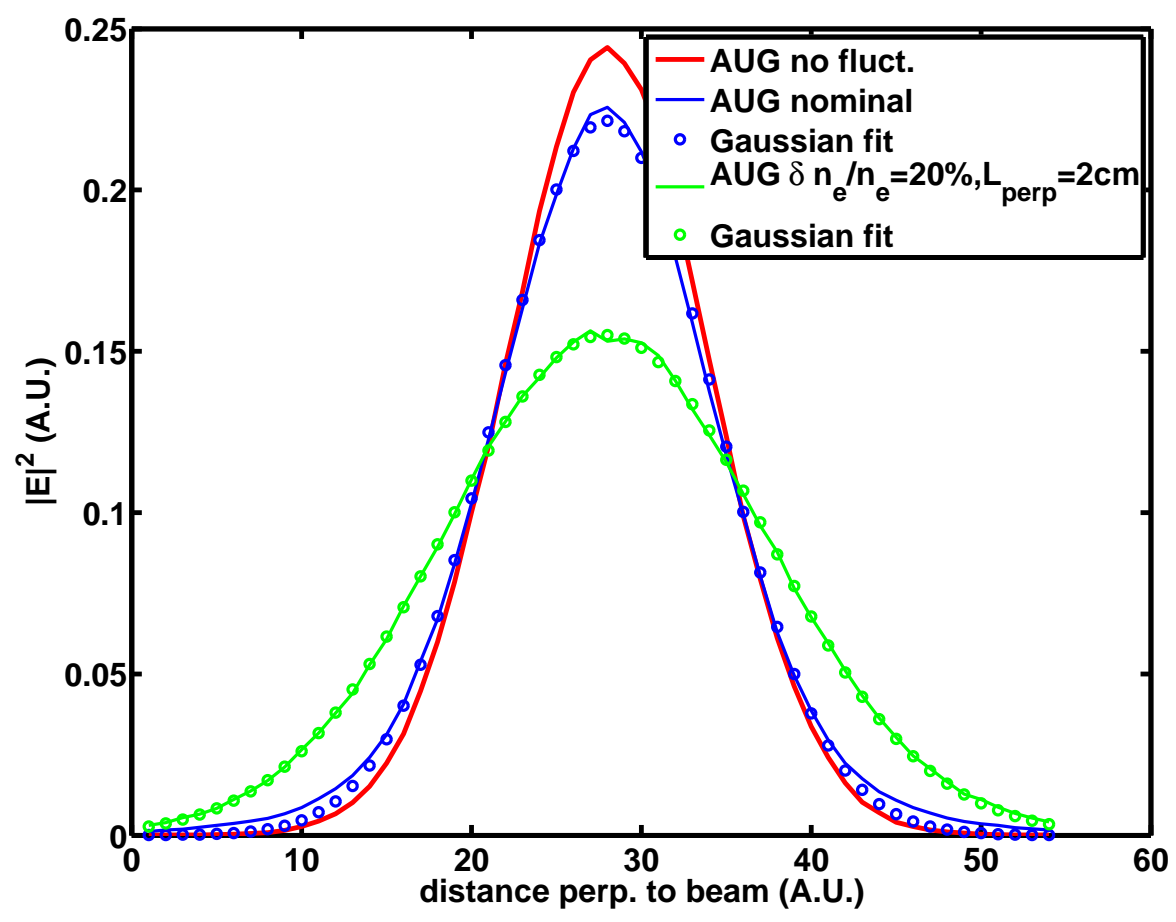

Figure 8. Shape of the EC beam after the fluctuation layer in AUG discharge \# 25485 with diffusive and superdiffusive parameters. Gaussian fit is suitable only for diffusive regime, while superdiffusive beam has characteristic high tails.

\section{Results for ITER electron cyclotron upper launcher}

\subsection{Simulated scenarios}

We have concentrated in this paper on the $15 \mathrm{MA} Q=10$ standard H-mode ITER scenario. It is the most important mission of ITER and thus it deserves special attention. Here we have modelled the flat-top phase according to the simulations presented in Ref. [33]‡. The relevant 1D profiles of electron density and temperature are shown in Figure 10 and flux surfaces of the equilibrium in Figure 11 (the distorted shape around the X-point is due to an equilibrium re-processing and does not affect the results in the top region, where the beam propagates).

Relevant parameters for the EC beams are given in Table 1. Four different configurations are listed, namely the upper steering mirror (USM) and lower steering mirror (LSM) and both are separately optimized for current drive at $q=2$ and $q=3 / 2$ surfaces, where the most dangerous NTMs are expected to occur in the given scenario.

$\ddagger$ The flat top phase of Case\#001 from Ref. [33] is used, which corresponds to the run with PPF file \# 53287/fkochl/jul2811/seq.1/ppfseq.17416. 


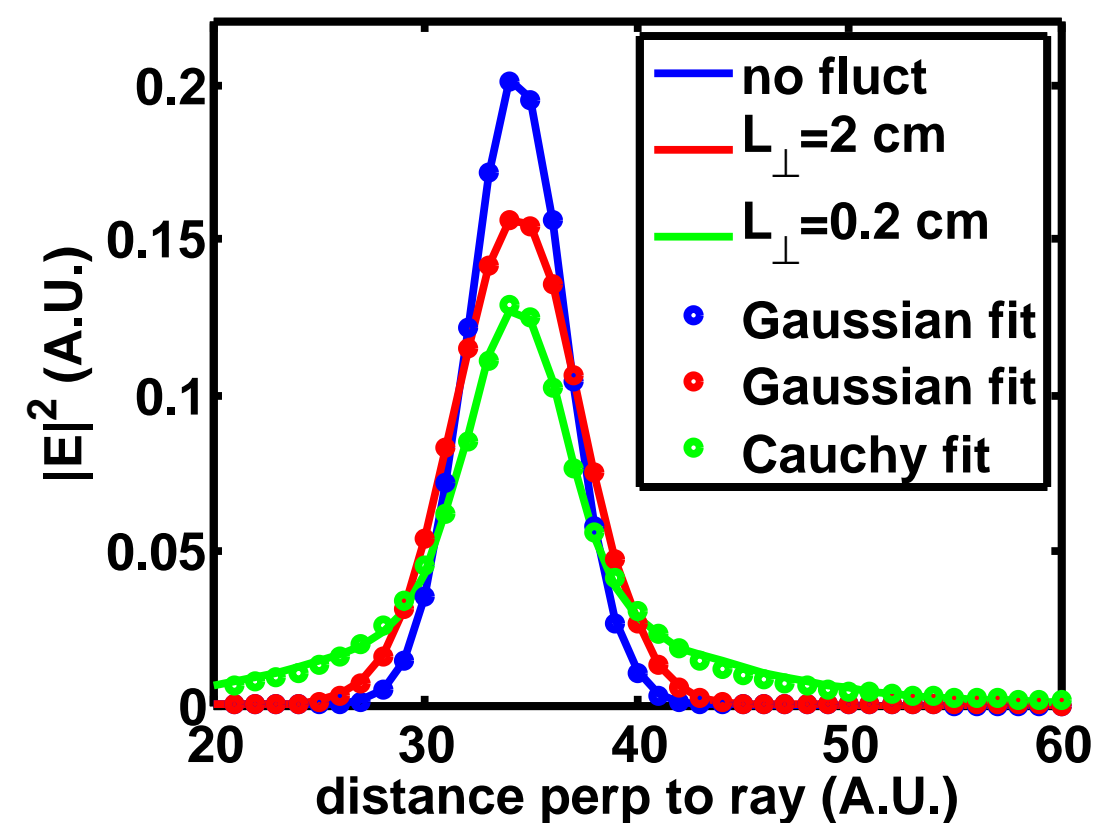

Figure 9. Shape of the EC beam after the fluctuation layer in ITER with diffusive and superdiffusive parameters. Gaussian fit is very accurately modeling the diffusive and quiescent beam shape, while Cauchy fit is necessary for super-diffusive parameters with elevated tails.

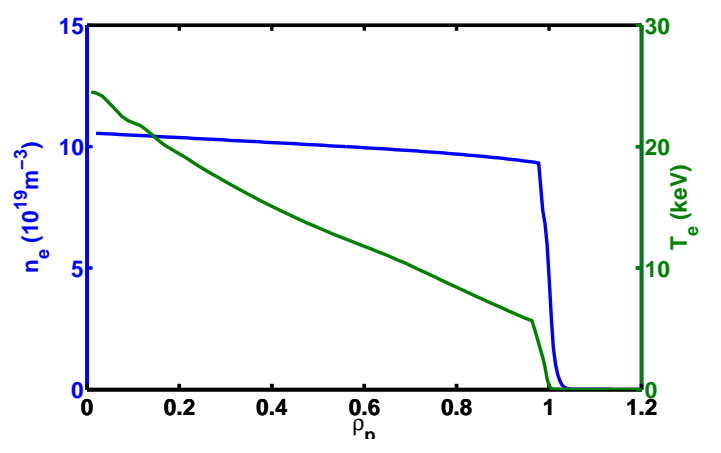

Figure 10. The kinetic profiles of electron density (left, solid) and temperature (right, dashed) in 15 MA ITER scenario.

\subsection{Absorption profiles and effect of ballooning}

In figure 12 we show the absorption profiles of electron cyclotron beams. The set of profiles on the left-hand side of the figure (around $\rho_{p}=0.77$ ) corresponds to deposition on the $q=3 / 2$ surface while the set on the right-hand side (around $\rho_{p}=0.87$ ) corresponds to the $q=2$ surface. Red/blue color represents the case with/without density fluctuations, respectively, while the solid line shows the USM configuration and the dashed line the LSM configuration. An immediate observation is that the beams are broadened by a factor of $\approx 3$ in all cases. Another peculiar feature of the 


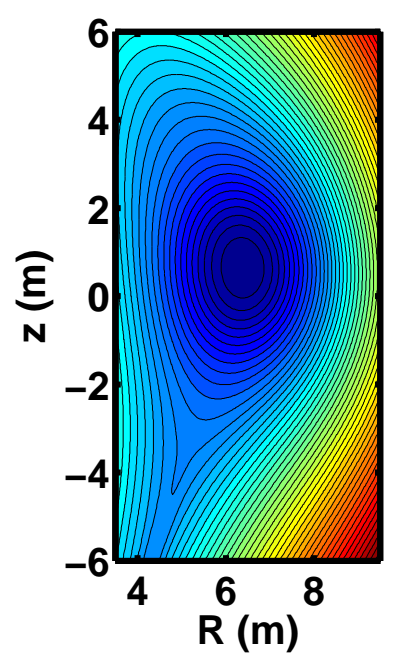

Figure 11. Flux surfaces of 15 MA standard H-mode scenario of ITER.

\begin{tabular}{|c|c|c|c|c|}
\hline Quantity & USM $(\mathrm{q}=2)$ & LSM $(\mathrm{q}=2)$ & USM $(\mathrm{q}=3 / 2)$ & LSM $(\mathrm{q}=3 / 2)$ \\
\hline Frequency $(\mathrm{GHz})$ & 170 & 170 & 170 & 170 \\
\hline Mode & $\mathrm{O}$ & $\mathrm{O}$ & $\mathrm{O}$ & $\mathrm{O}$ \\
\hline Input power $(\mathrm{MW})$ & 1 & 1 & 1 & 1 \\
\hline beam width $(\mathrm{cm})$ & 5.047 & 4.813 & 5.047 & 4.813 \\
\hline Focal length $(\mathrm{cm})$ & 318.6 & 200.1 & 318.6 & 200.1 \\
\hline Antenna R $(\mathrm{cm})$ & 699.9 & 705.4 & 699.9 & 705.4 \\
\hline Antenna z $(\mathrm{cm})$ & 441.4 & 417.8 & 441.4 & 417.8 \\
\hline Toroidal angle $(\mathrm{deg})$ & 20 & 20 & 20 & 20 \\
\hline Poloidal angle $(\mathrm{deg})$ & 46.8 & 40.5 & 54.7 & 50.1 \\
\hline Resonance surface $\left(\rho_{p}\right)$ & 0.87 & 0.87 & 0.77 & 0.77 \\
\hline
\end{tabular}

Table 1. Most important beam parameters for USM and LSM including both $(2,1)$ and $(3,2)$ resonance surfaces.

scattered profiles is the non-Gaussian shape. It can be explained by a combination of beam-broadening-induced geometrical factors and aberration effects (these are naturally included in the model as each ray carry information of the refractive index $N$ and also of the temperature that affects the absorption). The effect of density fluctuations is similar to both mirrors and both resonance surfaces, while the geometric distortion of the Gaussian shape is slightly stronger for inner $q=3 / 2$ surface compared to outer $q=2$. Each of these simulations was carried out using 400k-2M rays, which allows a statistically well-converged determination of the power deposition profiles.

One major uncertainty of the density fluctuations in open field lines is related to ballooning. In the core region with nested flux surfaces, the fluctuations are strongly damped at the high-field side but only very scarce information is available for the open field line region of $\mathrm{H}$-mode plasmas that is interesting for the present study. To evaluate 


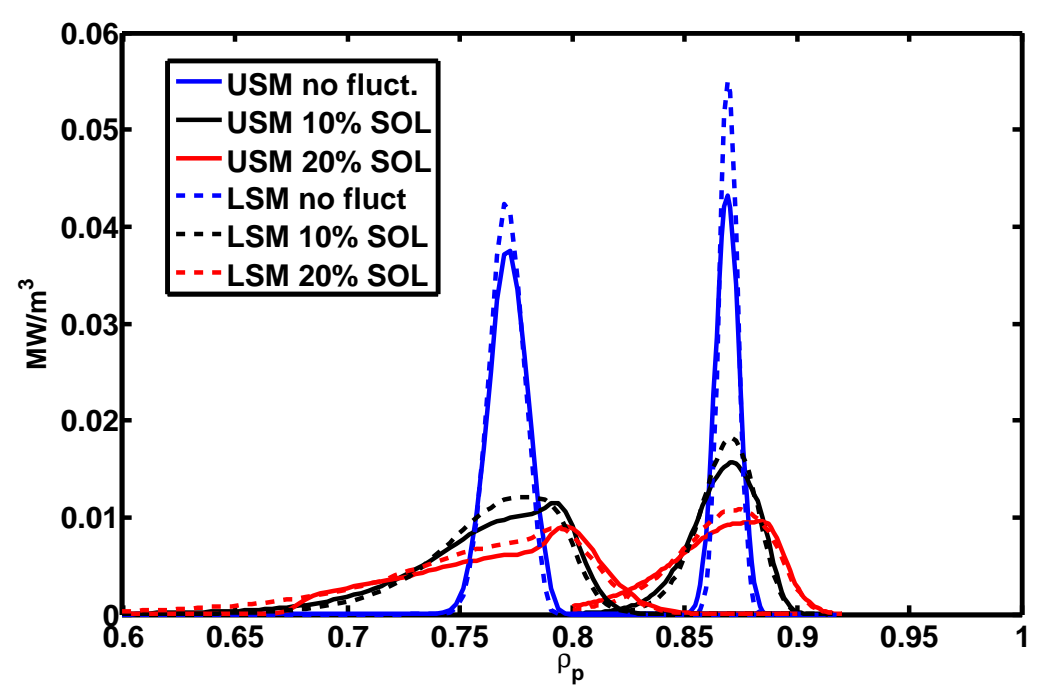

Figure 12. Absorption profiles for both mirrors and both resonance surfaces with and with out the scattering of density fluctuations.

the effect of ballooning on the profile broadening, we have assumed a core-like ballooning given by eq. (16). A simulation was carried out for USM $q=(2,1)$ configuration, assuming $20 \%$ fluctuation amplitude at the outer midplane. For comparison, the results are shown together with $20 \%$ and $10 \%$ fluctuation amplitudes without ballooning in Fig. 13. As expected, the ballooning case is very close to the $10 \%$ fluctuation amplitude case as the level of fluctuations at the location where the EC beam enters the plasma is roughly half of the midplane value, in this case $10 \%$.

\subsection{Fluctuation parameter scans}

Since some of the key input parameters have large uncertainties, a scan has been performed to assess the dependence of the results on these parameters. As a basis for this scan, we have selected USM configuration for the $q=2$ resonance surface. The scanned variables are the fluctuation amplitude $\delta n_{e} / n_{e}$ and the correlation length $L_{c}=L_{\perp}$ shown in figure 14. In this figure we show the full width at half-maximum (FWHM) of the deposition profile compared to the quiescent case. It is readily noticed that the effect of fluctuations is to broaden the beam by a factor of 1.7 to 5.1 , in the range of most likely fluctuation amplitudes by a factor of 2.5 to 3.5. In this scan no ballooning was assumed. In the case of correlation, inverse proportionality is observed. Different properties of the turbulence in the open-field-line region are mimicked through different values of the correlation length. An investigation of the different statistical properties of the edge turbulence requires a separate study and is left for future work. 


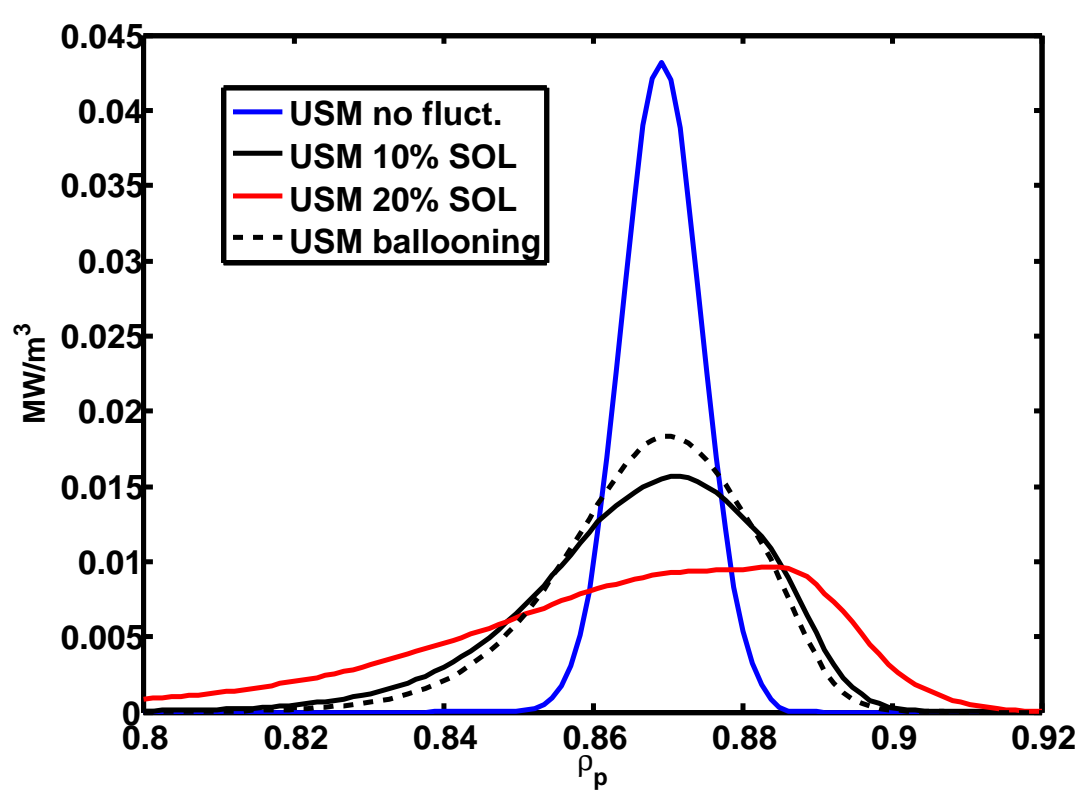

Figure 13. Absorption profiles with and without the ballooning in the density fluctuations.

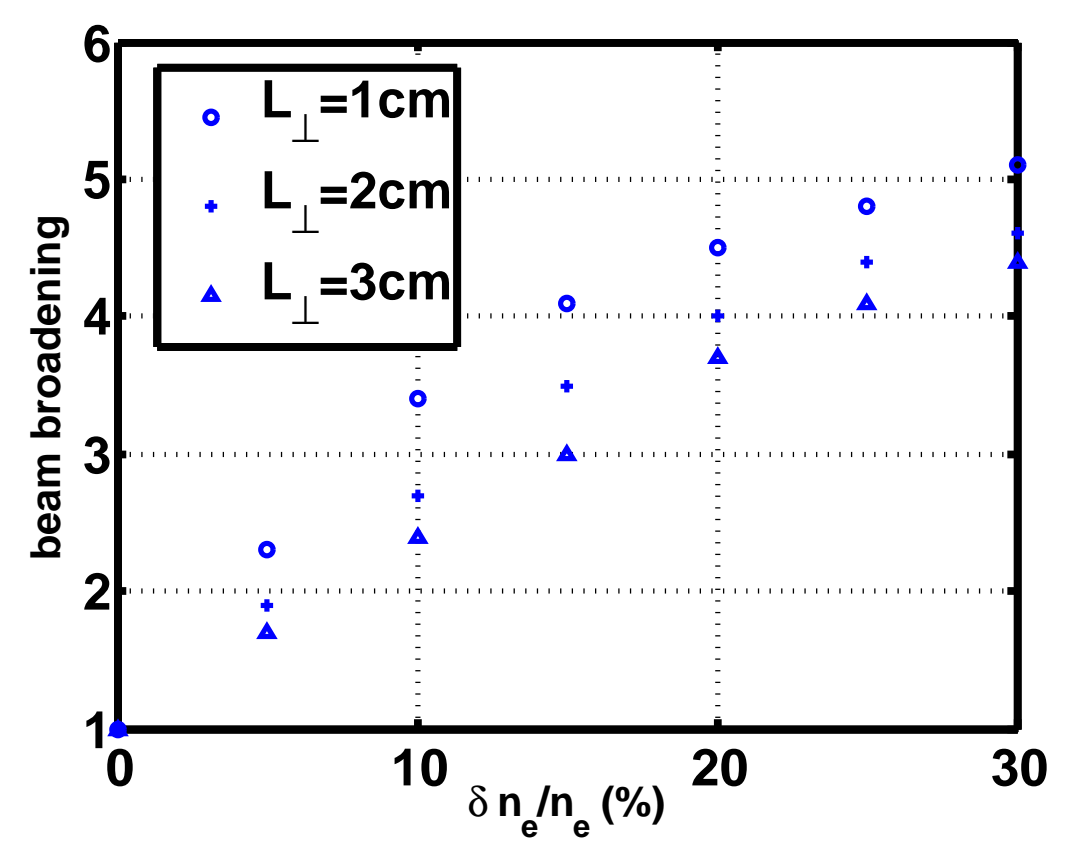

Figure 14. Beam broadening (FWHM of the deposition profile compared to the case without fluctuations) as a function of the fluctuation amplitude.

\section{Implications to ITER}

We have shown that using realistic parameters for the turbulence, the EC beam broadening is of the order of a factor of 2.5 to 3.5. In this paper we have only shown 
the broadening of the absorption profile while the NTM mitigation relies in the driven current inside the island. However, the driven current density can be directly linked to the absorption as [34]

$$
\langle j\rangle=\eta\left\langle\frac{d P}{d V}\right\rangle,
$$

where the current drive efficiency $\eta$ is a function of position. The broadening of the power profile applies also to the current density profile apart for the variation of $\eta$ across the deposition profile, which is usually negligible for localized EC deposition. A general formulation of the power threshold to mitigate NTMs is discussed e.g. in [9], which is largely based on the analyses of the generalized Rutherford equation presented in $[35,36]$. We may repeat the calculation assuming the beam driven current is broadened by factor of 1,2 or 3 . The resulting power is shown in figure 15 . The conclusions of [9] still remain: with the broadened beams continuos wave injection $(\mathrm{cw})$ demands more than the nominal power available in the EC UL $(13.3 \mathrm{MW}$ per row of mirrors, $\approx 20 \mathrm{MW}$ in total [9]), while the modulated (mod) power is still managable. Although in this case the whole UL might need to be reserved for NTM suppression only.

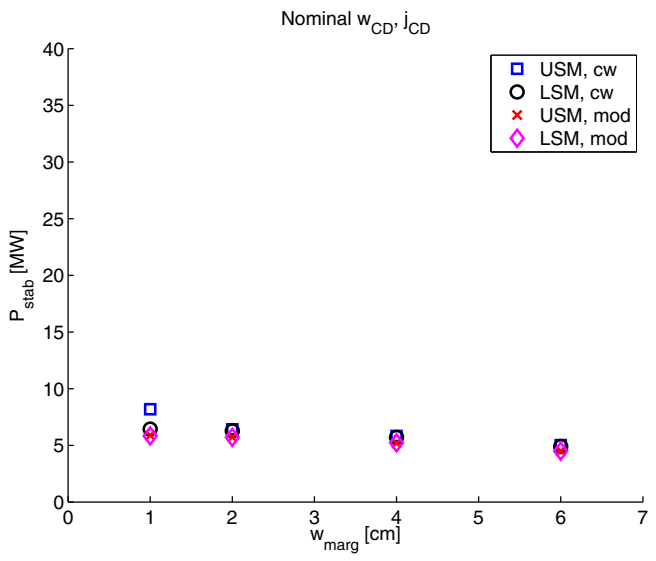

a) b)

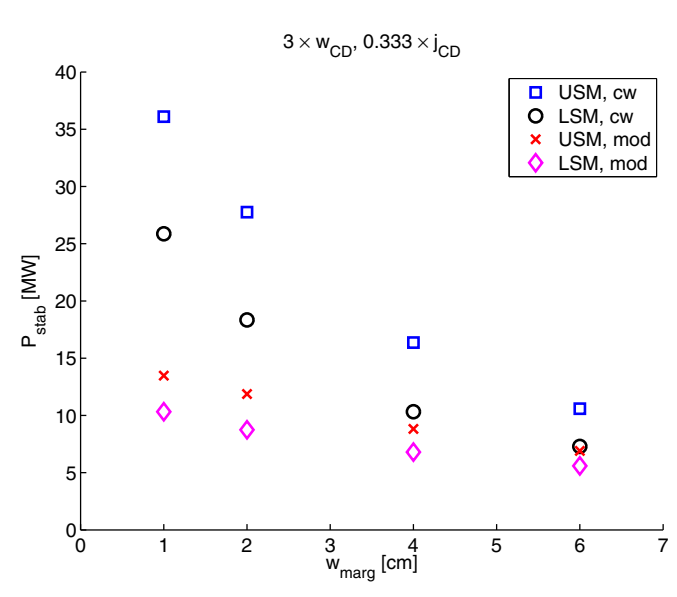

Figure 15. Power required for complete $(2,1)$ NTM suppression as a function of marginal island width for beam with nominal power deposition profile a), broadened by a factor of 3 . Similar figure with the broadening by a factor of two was published in [9].

\section{Summary}

To summarize, in this paper we have presented simulations of electron cyclotron wave propagation and absorption in ITER, retaining the effects of scattering due to edge density fluctuations. This is particularly important for the upper launcher of ITER because of NTM stabilization. The simulations were carried out using WKBeam code that was benchmarked against paraxial beam-tracing code TORBEAM and full-wave 
IPF-FDMC code. In particular, we showed that the Born approximation utilized in the WKBeam code is applicable in the parameter range of ITER. We analyzed the scattering operator which describes the effects of turbulence on the wave, showing that the transport due to density fluctuation can be of diffusive or superdiffusive nature. Moreover, it was shown that nominal parameters for ASDEX Upgrade tokamak lie barely on the superdiffusive regime while ITER is deeply on the diffusive regime. Because of this and because of the larger machine size, a larger beam broadening can be expected in ITER.

The input needed for the averaged scattering includes statistical averages over the turbulence, namely the fluctuation amplitude $\delta n_{e} / n_{e}$, that was assumed to be a function of radial coordinate $\rho_{p}$ and the poloidal geometrical angle $\theta$, and the perpendicular correlation length $L_{\perp}$. Based on our present knowledge, and "educated guess" for the value of these quantities in the ITER 15 MA standard H-mode scenario has been discussed. Extensive scans have been performed in order to assess the sensitivities of the results to the relevant parameters. Thereafter, simulations were carried out to calculate the beam broadening defined as a increase of the FHWM of the absorption profile. Broadening by a factor of 1.7 to 5.1 was observed depending on the turbulence parameters. In the most likely regime of parameters a broadening by a factor of 2.5 to 3.5 is found. It was observed that the ballooning like poloidal dependence of the fluctuation amplitude decreases the broadening to levels found with half of the fluctuation amplitude without the ballooning, leading to roughly a decrease of broadening by a factor of 2 . Parameter scans were shown for the most important turbulence parameters, i.e. the fluctuation level in the SOL and the correlation length. Last but not least, it was shown that, even with a factor of 3 broadening, NTM mitigation by ECCD in ITER is still plausible but modulation of EC power could become necessary to achieve NTM suppression within the available power.

\section{Outlook}

In this paper we concentrated on the most important ITER mission, i.e. the $15 \mathrm{MA}$ standard H-mode and only in flat-top phase. The next steps include similar analysis in other time slices and scenarios. Moreover, ITER is equipped with equatorial launcher (EL) that is foreseen to be mostly used for plasma heating and control applications. Some recent work has been carried out to investigate whether this system could be used to drive current in the $q=1$ surface to destabilize sawtooth [6, 37]. Therefore, the work will be extended to EL as well. Although in this paper we considered only beam broadening effects, the mode-to-mode scattering should not be forgotten. It is important both for NTM mitigation, as part of the power is not coupled to plasma, and to stray radiation studies. We expect mode-to-mode scattering to be important also for EL. Recent numerical development of WKBeam code aims to generalize the input magnetic field for full 3D geometry. This enables to study scattering phenomena in stellarators, in

particular W7-X that is equipped with good diagnostics possible capable of measuring 
beam broadening. Furthermore, larger scale turbulent blobs and ELM structures might affect the propagation of EC waves. Future work to estimate this effect in terms of NTM mitigation is foreseen.

\section{Acknowledgements}

This work has been performed within Fusion for Energy Grant 615. The views expressed in this publication are the sole responsibility of the author and do not necessarily reflect the views of Fusion for Energy.

\section{References}

[1] R J Buttery, S Günter, G Giruzzi, T C Hender, D Howell, G Huysmans, R J La Haye, M Maraschek, H Reimerdes, O Sauter, C D Warrick, H R Wilson, and H Zohm. Neoclassical tearing modes. Plasma Physics and Controlled Fusion, 42(12B):B61, 2000.

[2] G. Gantenbein, H. Zohm, G. Giruzzi, S. Günter, F. Leuterer, M. Maraschek, J. Meskat, Q. Yu, ASDEX Upgrade Team, and ECRH-Group (AUG). Complete suppression of neoclassical tearing modes with current drive at the electron-cyclotron-resonance frequency in asdex upgrade tokamak. Phys. Rev. Lett., 85:1242-1245, Aug 2000.

[3] R.J. La Haye, A. Isayama, and M. Maraschek. Prospects for stabilization of neoclassical tearing modes by electron cyclotron current drive in iter. Nuclear Fusion, 49(4):045005, 2009.

[4] L. Urso, H. Zohm, A. Isayama, M. Maraschek, E. Poli, ASDEX Upgrade Team, and JT-60 Team. ASDEX Upgradejt-60u comparison and ecrh power requirements for ntm stabilization in iter. Nuclear Fusion, 50(2):025010, 2010.

[5] M A Henderson et al. Fusion Engineering and Design, 82:454, 2007.

[6] M.A. Henderson, R. Heidinger, D. Strauss, R. Bertizzolo, A. Bruschi, R. Chavan, E. Ciattaglia, S. Cirant, A. Collazos, I. Danilov, F. Dolizy, J. Duron, D. Farina, U. Fischer, G. Gantenbein, G. Hailfinger, W. Kasparek, K. Kleefeldt, J.-D. Landis, A. Meier, A. Moro, P. Platania, B. Plaum, E. Poli, G. Ramponi, G. Saibene, F. Sanchez, O. Sauter, A. Serikov, H. Shidara, C. Sozzi, P. Spaeh, V.S. Udintsev, H. Zohm, and C. Zucca. Overview of the iter ec upper launcher. Nuclear Fusion, 48(5):054013, 2008.

[7] R. Heidinger, R. Bertizzolo, A. Bruschi, R. Chavan, S. Cirant, A. Collazos, M. de Baar, B. Elzendoorn, D. Farina, U. Fischer, J. Gafert, F. Gandini, G. Gantenbein, A. Goede, T. Goodman, G. Hailfinger, M. Henderson, W. Kasparek, K. Kleefeldt, J.-D. Landis, A. Meier, A. Moro, P. Platania, E. Poli, G. Ramponi, G. Saibene, F. Sanchez, O. Sauter, T. Scherer, A. Serikov, H. Shidara, C. Sozzi, P. Spaeh, D. Strauss, V.S. Udintsev, A. Vaccaro, H. Zohm, and C. Zucca. Conceptual design of the $\{\mathrm{ECH}\}$ upper launcher system for $\{\mathrm{ITER}\}$. Fusion Engineering and Design, 84(26):284 - 289, 2009. Proceeding of the 25th Symposium on Fusion Technology(SOFT-25).

[8] M. Henderson, G. Saibene, C. Darbos, D. Farina, L. Figini, M. Gagliardi, F. Gandini, T. Gassmann, G. Hanson, A. Loarte, T. Omori, E. Poli, D. Purohit, and K. Takahashi. The targeted heating and current drive applications for the iter electron cyclotron system. Physics of Plasmas, 22(2), 2015.

[9] E. Poli, C. Angioni, F.J. Casson, D. Farina, L. Figini, T.P. Goodman, O. Maj, O. Sauter, H. Weber, H. Zohm, G. Saibene, and M.A. Henderson. On recent results in the modelling of neoclassicaltearing-mode stabilization via electron cyclotron current drive and their impact on the design of the upper ec launcher for iter. Nuclear Fusion, 55(1):013023, 2015.

[10] M. W. Brookman, M. E. Austin, and C. C. Petty. Finding evidence for density fluctuation effects 
on electron cyclotron heating deposition profiles on diii-d. AIP Conference Proceedings, 1689(1), 2015.

[11] Christos Tsironis, Arthur G. Peeters, Heinz Isliker, Dafni Strintzi, Ioanna Chatziantonaki, and Loukas Vlahos. Electron-cyclotron wave scattering by edge density fluctuations in iter. Physics of Plasmas, 16(11), 2009.

[12] Yves Peysson, Joan Decker, L Morini, and S Coda. Rf current drive and plasma fluctuations. Plasma Physics and Controlled Fusion, 53(12):124028, 2011.

[13] A Köhn, E Holzhauer, J Leddy, M B Thomas, and R G L Vann. Influence of plasma turbulence on microwave propagation. Plasma Physics and Controlled Fusion, 58(10):105008, 2016.

[14] Weber, Hannes, Maj, Omar, and Poli, Emanuele. Scattering of diffracting beams of electron cyclotron waves by random density fluctuations in inhomogeneous plasmas. EPJ Web of Conferences, 87:01002, 2015.

[15] L. Guidi, O. Maj, H. Weber, A. Köhn, A. Snicker, and E. Poli. Cross-polarization scattering of diffracting electron-cyclotron beams in a turbulent plasma with the wkbeam code. Journal of Physics: Conference Series, 775(1):012005, 2016.

[16] Steven W. McDonald. Wave kinetic equation in a fluctuating medium. Phys. Rev. A, 43:44844499, Apr 1991.

[17] Frank C. Karal and Joseph B. Keller. Elastic, electromagnetic, and other waves in a random medium. Journal of Mathematical Physics, 5(4):537-547, 1964.

[18] Steven W. McDonald. Phase-space representations of wave equations with applications to the eikonal approximation for short-wavelength waves. Physics Reports, 158(6):337 - 416, 1988.

[19] I B Bernstein. Physics of Fluids, 18:320, 1975.

[20] Yu A Kravtsov and Yu I Orlov. Geometrical Optics of Inhomogeneous Media. Springer Verlag, Berlin, 1990.

[21] M. Bornatici et al. Electron cyclotron emission and absorption in fusion plasmas. Nuclear Fusion, 23(9):1153, 1983.

[22] D. Farina. A quasi-optical beam-tracing code for electron cyclotron absorption and current drive: Gray. Fusion Science and Technology, 52(2):154-160, 2007.

[23] E. Poli, A.G. Peeters, and G.V. Pereverzev. Torbeam, a beam tracing code for electron-cyclotron waves in tokamak plasmas. Computer Physics Communications, 136(1):90 - 104, 2001.

[24] H Weber. IPP Report 5/134, 2013.

[25] S J Zweben, J A Boedo, O Grulke, C Hidalgo, B LaBombard, R J Maqueda, P Scarin, and J L Terry. Edge turbulence measurements in toroidal fusion devices. Plasma Physics and Controlled Fusion, 49(7):S1, 2007.

[26] G D Conway. Turbulence measurements in fusion plasmas. Plasma Physics and Controlled Fusion, 50(12):124026, 2008.

[27] C Silva, H Figueiredo, P Duarte, and H Fernandes. Characterization of the poloidal asymmetries in the isttok edge plasma. Plasma Physics and Controlled Fusion, 53(8):085021, 2011.

[28] G.R. Tynan, J.A. Boedo, D.S. Gray, R. Van Nieuwenhove, G. Van Oost, and R.R. Weynants. Effects of radial electric fields on the turbulence and transport in the textor edge and sol plasma. Journal of Nuclear Materials, 196:770 - 774, 1992.

[29] T.L. Rhodes, R.J. Taylor, E.J. Doyle, Jr. N.C. Luhmann, and W.A. Peebles. Poloidally asymmetric response of turbulence to the h mode on the cct tokamak. Nuclear Fusion, 33(12):1787, 1993.

[30] A Köhn, Cappa, E Holzhauer, F Castejón, Fernández, and U Stroth. Full-wave calculation of the oxb mode conversion of gaussian beams in a cylindrical plasma. Plasma Physics and Controlled Fusion, 50(8):085018, 2008.

[31] V. Zaburdaev, S. Denisov, and J. Klafter. Lévy walks. Rev. Mod. Phys., 87:483-530, Jun 2015.

[32] V. Pierrard and M. Lazar. Kappa distributions: Theory and applications in space plasmas. Solar Physics, 267(1):153-174, 2010.

[33] V Parail et al. Nuclear Fusion, 53:113002, 2013.

[34] T M Antonsen and K R Chu. Physics of Fluids, 25:1295, 1982. 
[35] H Zohm et al. Plasma Physics and Controlled Fusion, 49:B341, 2007.

[36] O Sauter et al. Plasma Physics and Controlled Fusion, 52:025002, 2010.

[37] I.T. Chapman, J.P. Graves, O. Sauter, C. Zucca, O. Asunta, R.J. Buttery, S. Coda, T. Goodman, V. Igochine, T. Johnson, M. Jucker, R.J. La Haye, M. Lennholm, and JET-EFDA Contributors. Power requirements for electron cyclotron current drive and ion cyclotron resonance heating for sawtooth control in iter. Nuclear Fusion, 53(6):066001, 2013. 